\section{Welche Analgetika bei neuropathischen Schmerzen?}

\section{Zwischen klinischen Studien und dem praktischen Alltag klaffen oft große Lücken. Während Metaanalysen eher von Opioiden abraten, geben die Leit- linien gegensätzliche Empfehlungen. Sind solch abweichende Ergebnisse möglicherweise die Folge fehlender Praxisnähe der Studien?}

$E_{\text {ith }}^{\text {th }}$ twa $15,5 \%$ aller Schmerzen sind in - ihrem Ursprung als neuropathisch einzuordnen [1]. Bezüglich der optimalen Therapie bei diesen Schmerzen machte im vergangenen Jahr die Kommunikation einer in Lancet Neurology publizierte Metaanalyse von sich reden [2]. Auf Basis der Auswertung von 229 randomisierten, placebokontrollierten Doppelblindstudien wurden von den Autoren als orale oder topische Erstlinientherapie trizyklische Antidepressiva (TCA), Serotonin-Noradrenalin-Wiederaufnahmehemer (SNRI) sowie Pregabalin und Gabapentin empfohlen. Als Zweitlinientherapie wurden Lidocainoder Capsaicin-Pflaster oder das schwache Opioid Tramadol genannt, erst in dritter Linie wurde eine „schwache Empfehlung “ für starkwirksame Opioide ausgesprochen.

\section{Widerspruch durch falsche Interpretation}

Dies widerspricht nicht nur klinischer Erfahrung, sondern auch der bekannten S3-Leitlinie zur Langzeitanwendung von Opioiden bei nicht tumorbedingten Schmerzen (LONTS [3]), konstatierte Privatdozent Dr. Michael Überall aus Nürnberg. Seine Analyse der Originalpublikation ergab deutliche Schwächen der Daten sowie deren fehlerhafte Interpretation. Hinsichtlich der „number needed to treat" (NNT) für eine $50 \%$-ige Schmerzlinderung seien nämlich TCA und starke sowie schwache Opioide mit NNT zwischen drei und fünf den anderen Substanzen mit NNT zwischen sechs und acht mindestens ebenbürtig oder sogar überlegen. Die positive Einschätzung der Opioide bei neuropathischen Schmerzen spiegele sich auch in den Empfehlungen der LONTS-Leitlinien und den aktuellen DGN-Leitlinien wider, sagte Überall. Dem widerspricht wiederum eine Untersuchung, in der re- tardiertes Tapentadol neuropathische Schmerzen besser linderte als die Kombination aus Oxycodon und Naloxon [4]. Fragen werfe diese letztere Untersuchung jedoch wegen ihres ,alltagsfernen Szenarios" und hoher Abbruchraten von über $50 \%$ auf, so der Schmerzmediziner.

\section{Alltagsnahe Studien besonders wichtig}

Ein praxisgerechtes Studiendesign bestehe für Überall in einer offenen Therapie unter realistischen Alltagsbedingungen mit patientenrelevanten, gemessenen Endpunkten und der Minimierung externer Einflüsse. Eine solche Studie müsse weiterhin eine Dosisfindung nach individueller Befindlichkeit und eine verblindete Endpunktanalyse aufweisen. „Man kann heute aus dem Alltag durchaus Daten generieren, mit denen man auch im Alltag arbeiten kann", argumentierte Überall.

In einer eigenen Studie hat er die Wirksamkeit von Oxycodon/Naloxon mit Tapentadol bei Patienten mit länger bestehenden Rückenschmerzen neuropathischer Genese verglichen [5]. Fast alle Patienten waren mit WHO-Stufe-IAnalgetika vorbehandelt. Über zwölf Behandlungswochen fand sich in beiden Gruppen eine statistisch nicht signifikant unterschiedliche deutliche Abnahme des Schmerzindex auf der visuellen Analogskala (96,9\% unter Oxycodon/ Naloxon vs. $61,6 \%$ unter Tapentadol). Diese Medikamente „sind für uns als Praktiker vergleichbar wirksam." Mit dem Ausmaß der erzielten Schmerzlinderung nahm jedoch der Unterschied der Response im zeitlichen Verlauf zu. Während bei einer $30 \%$-igen Abnahme der Schmerzintensität kein Unterschied zwischen den Behandlungsgruppen feststellbar war, änderte sich dies mit zunehmendem Ausmaß der Besserung: Eine hochsignifikante Schmerzreduktion um
$70 \%$ fand sich nach zwölf Wochen bei etwa $15 \%$ der Patienten unter Tapentadol, aber bei knapp $40 \%$ unter dem Opioid. Das bedeute ,je härter das Wirksamkeitskriterium für die analgetische Wirkung ist, umso eher sehen wir einen Unterschied in der Therapie“, erläuterte Überall. Damit einher ging in beiden Therapiegruppen eine Abnahme der als rein neuropathisch definierten Schmerzen hin zu nozizeptiven Schmerzen und dem „mixed pain“-Phänotyp.

Opioidantagonisten vorteilhaft für anspruchsvolle Therapie

Ähnliche Vorteile wie hinsichtlich der Schmerzintensität fand sich für Oxycodon/Naloxon in den Gruppen mit höherer Schmerzreduktion (um $70 \%$ ) auch für die Parameter Funktionalität und Lebensqualität. Keinen Unterschied zwischen beiden Medikamenten gab es in puncto Verträglichkeit unter Alltagsbedingungen. Erstaunlicherweise gab es auch bei dem Opioid-typischen Parameter Stuhlverhalten/Darmfunktion keine Differenzen, wie Überall hervorhob.

Ein zusammengesetzter Endpunkt aus allen Wirksamkeits- und Verträglichkeitsparametern wurde bei 4 von 10 Patienten unter Oxycodon/Naloxon und von 2,5 von 10 Patienten unter Tapentadol erreicht.

Überalls Fazit: Beide Medikamente seien bei neuropathischen Schmerzen gut wirksam und gut verträglich, aber „sobald die Ansprüche an die Therapie ein bisschen steigen, wir mehr analgetische Wirkung, mehr Verbesserung haben wollen, haben wir den deutlich messbaren Vorteil mit dem Opioidagonisten."

Dr. Andreas Häckel

\section{Literatur}

1. Erhebung der Techniker Krankenkasse

2. Finnerup NB et al. Lancet Neurol 2015; 14(2): 162-73

3. Häuser W et al. Deutsches Ärzteblatt 2014; 111(432):732-40

4. Baron R et al. Pain Practice (2015): DOI 10.1111/papr.12308

5. Überall MA, Müller-Schwefe GHH. J Pain Research 2016; submitted for publication

Symposium "Sinn und Unsinn von WHO-StufeIII-Analgetika bei chronischen neuropathischen Schmerzen", Frankfurt am Main, 4.3.2016 